\title{
Détermination des priorités en chirurgie pédiatrique spécialisée au Canada durant la pandémie de COVID-19
}

\author{
Erik D. Skarsgard MSc MD, pour les Pediatric Surgical Chiefs of Canada*
}

- Citation : CMAJ 2020 October 13;192:E1212-3. doi : 10.1503/cmaj.201577-f; diffusion hâtive le $1^{\text {er }}$ septembre 2020

Voir la version anglaise de l'article ici : www.cmaj.ca/lookup/doi/10.1503/cmaj.201577

*La liste complète des membres du groupe se trouve à la fin de l'article.

$\mathbf{L}$ e 11 mars 2020, l'Organisation mondiale de la Santé a déclaré que l'épidémie de maladie à coronavirus 2019 (COVID-19) avait atteint le statut de pandémie. Les gouvernements des provinces et des territoires ont alors commencé à préparer les hôpitaux à une envolée des hospitalisations et des admissions aux soins intensifs en lien avec la maladie. Les établissements hospitaliers se sont mis à annuler les interventions chirurgicales planifiées; selon les estimations, le taux d'annulation à la fin mars avoisinait les 33000 par semaine ${ }^{1}$. Quel a été l'effet de ces politiques sur le milieu de la chirurgie pédiatrique surspécialisée canadien? Et comment reprendre le retard accumulé?

À l'aide d'infrastructures adaptées aux enfants, des chirurgiens pédiatriques surspécialisés, des anesthésiologistes, des pédiatres, des infirmières en pédiatrie et d'autres professionnels de la santé prodiguent des soins chirurgicaux dans 15 hôpitaux pour enfants au Canada. Les hôpitaux pour enfants sont des centres régionaux de soins tertiaires et quaternaires en chirurgie : c'est là que l'on opère les enfants qui ont une cardiopathie congénitale, une tumeur au cerveau ou à un organe plein, une scoliose, une affection qui compromet l'audition ou la vision, ou des problèmes médicaux complexes. Les greffes cardiaques et d'organe plein sont des services régionalisés, entre l'est et l'ouest du pays. Les personnes de 17 ans et moins subissent environ $5 \%$ des interventions chirurgicales pratiquées chez des patients hospitalisés, selon les données de l'Institut canadien d'information sur la santé ${ }^{2}$. Cela dit, environ la moitié de ces interventions - dont l'appendicectomie, la réparation herniaire, la pose de tubes transtympaniques et l'amygdalectomie avec adénoïdectomie - sont effectuées chez des enfants plus âgés généralement en santé, dans des hôpitaux non pédiatriques, par des chirurgiens qui opèrent surtout des adultes. Elles ne seront donc pas abordées dans le présent article.

\section{POINTS CLÉS}

- Les enfants canadiens nécessitant une opération spécialisée planifiée sont pris en charge par une équipe chirurgicale surspécialisée dans 1 des 15 hôpitaux pédiatriques du pays, mais doivent, comme les adultes, attendre leur tour.

- Les hôpitaux pour enfants du Canada, comme les autres hôpitaux, ont participé à la lutte systémique contre la maladie à coronavirus 2019 (COVID-19) en annulant des interventions chirurgicales planifiées pour opérer en priorité les cas urgents, et en élaborant des politiques et des protocoles visant à réduire le risque de transmission du coronavirus du syndrome respiratoire aigu sévère 2 (SRAS-CoV-2) chez les patients et le personnel.

- Maintenant que les hôpitaux reprennent les interventions planifiées, ils doivent se doter, pour les opérations pédiatriques reportées, d'un cadre de définition des priorités et d'action qui reflète les besoins uniques des enfants par rapport à ceux des adultes et qui favorise l'accès aux soins chirurgicaux des familles déjà désavantagées par des inégalités sociales et économiques.

Le domaine de la chirurgie pédiatrique est souvent négligé au Canada ${ }^{3}$. Les interventions planifiées (aussi appelées électives) chez les enfants suivent des délais d'attente cibles (allant de moins de 24 heures à moins de 12 mois) établis par consensus national, ce qui permet une évaluation et une déclaration fiables du rendement sur le plan des interventions effectuées 4 . Les données de 2018 révèlent qu'au Canada, seulement $65 \%$ des opérations planifiées dans les hôpitaux pour enfants ont été réalisées dans les délais prévus, ce qui laisse croire que la capacité nationale était déjà insuffisante avant la pandémie ${ }^{5}$.

Au début des préparatifs en vue de la hausse rapide des cas de COVID-19, il a été proposé d'envoyer des personnes majeures dans des hôpitaux pédiatriques. Cette solution n'a pas été 
nécessaire, sauf pour 3 adultes québécois atteints de la maladie, dont 2 ont dû être mis sous ventilation mécanique (Pramod Puligandla, Service de chirurgie pédiatrique, Hôpital de Montréal pour enfants, Centre universitaire de santé McGill, Montréal, Québec : communication personnelle, 2020). L’hospitalisation d'adultes n'a pas interrompu la prestation de services pour les enfants et n'a pas eu de répercussions négatives pour le personnel soignant. Les interventions chirurgicales d'urgence (non planifiées) n'ont jamais cessé pendant la crise; les interventions planifiées, quant à elles, ont été limitées à celles jugées prioritaires, entre la semaine du 16 mars 2020 et la fin mai ou le début juin, selon la province. Conséquemment, le nombre d'opérations chez les enfants a abruptement chuté au pays. Les interventions prioritaires comprenaient notamment les opérations au cœur ou pour un cancer, et, après quelques semaines, les greffes d'organe plein, les opérations au délai d'attente cible court, les opérations sur des patients symptomatiques et les opérations qui sont prévues à un stade développemental précis et dont le report nuirait à la capacité fonctionnelle (comme la spondylodèse, pour les cas de scoliose). Bien que près de 9000 interventions d'urgence et prioritaires aient été pratiquées dans les hôpitaux pour enfants du Canada entre la mi-mars et le mois de juin, on estime qu'il y a eu 7600 reports d'opérations, et 4000 noms d'enfant non ajoutés à la liste d'attente en raison d'un accès restreint à la consultation (Helene Flageole, Hôpital pour enfants McMaster et Pediatric Surgical Chiefs of Canada, Hamilton, Ontario : communication personnelle, 2020).

Pour reprendre le retard accumulé, il faudra augmenter la capacité d'exécution d'interventions planifiées (en prolongeant les horaires de chirurgie de semaine et de fin de semaine, en ouvrant des salles supplémentaires, etc.), gonfler les effectifs (infirmières, anesthésiologistes et chirurgiens) et voir un engagement pour le bien-être au travail. Nous aurons aussi besoin d'équipement spécialisé, de médicaments, de services diagnostiques (surtout de laboratoire et en radiologie) et d'équipement de protection individuelle en quantités suffisantes. Comme plusieurs provinces connaissent déjà une pénurie d'infirmières en pédiatrie et d'anesthésiologistes, le recrutement et la rétention des médecins en fonction des besoins et la formation du personnel infirmier directement dans l'établissement seront essentiels au rattrapage. La priorité devrait être accordée aux opérations repoussées en raison de la COVID-19 et aux opérations au délai d'attente cible court, dont le report augmente le risque de méfaits (p. ex. pour une hernie incarcérée chez un nouveau-né). Certaines atteintes (telles que la scoliose et l'épilepsie) progressant à des rythmes différents, des patients qui en sont atteints pourraient devoir passer devant les autres. De plus, l'affectation des salles d'opération aux différentes spécialités devrait se faire selon la longueur des listes d'attente, et accorder plus de temps aux spécialités dont les délais d'intervention dépassent les cibles nationales.

La pandémie a allongé les processus périopératoires (point à prendre en compte dans les projections de volume) et a limité l'accès aux cliniques de soins ambulatoires. Le rattrapage du retard en chirurgie passera par une accessibilité suffisante des évaluations pré- et postopératoires et par de nouvelles recommandations, qui peuvent en grande partie se faire à distance. Le réaménagement et la réorganisation des cliniques de soins ambulatoires de sorte à réduire l'attente, à rendre possible l'éloignement physique, à intégrer le dépistage et la préparation des patients et à faire les suivis virtuellement lorsque possible peuvent recentrer l'expérience de soins sur le patient, un résultat qu'il faudrait faire perdurer après la pandémie.

Même si les cas de COVID-19 dans la population pédiatrique semblaient initialement peu nombreux, de nouvelles données indiquent que le tiers des enfants qui contractent le coronavirus du syndrome respiratoire aigu sévère 2 (SRAS-CoV-2) seraient asymptomatiques ${ }^{6}$, et que la sensibilité des tests de réaction en chaîne par polymérase qui servent à en détecter l'ARN dans des prélèvements sur écouvillon serait inférieure à $80 \%$ chez les enfants ${ }^{7}$. Comme on a l'impression qu'un adulte est plus susceptible qu'un enfant du même ménage de transmettre le virus, les hôpitaux pour enfants ont limité le nombre d'adultes accompagnateurs autorisés. Même les hôpitaux qui d'ordinaire encourageaient la présence des parents en salle d'opération pour la pratique de l'anesthésie se sont mis à l'autoriser seulement dans certaines circonstances, une restriction qui dans bien des cas perdure à ce jour. Il faut toutefois envisager l'accès aux soins chirurgicaux différemment pour les familles désavantagées par l'éloignement géographique, l'instabilité économique et d'autres facteurs, pour lesquelles les cliniques virtuelles et communautaires devront être maintenues, voire multipliées. L'adaptation des communications à la culture, entre autres avec les communautés autochtones, sera essentielle.

Au Canada, les patients doivent souvent franchir des frontières pour subir une opération cardiaque, une greffe ou certaines interventions à la moelle épinière ou interventions à effraction minimale qui ne sont pas effectuées dans toutes les provinces. Dans ce contexte, les risques pour la santé publique que posent les déplacements doivent faire l'objet de mesures d'atténuation. La situation nécessite aussi, comme quelques provinces l'ont déjà fait, l'adoption d'un cadre éthique visant à résoudre les différends dans la détermination des cas prioritaires parmi les enfants des différentes provinces. Les mineurs qui attendent d'être opérés dans un hôpital général risquent d'être oubliés dans le raz-de-marée d'adultes dont l'intervention a été reportée. Nous devons faire appel à nos collègues chirurgiens qui travaillent avec la population adulte pour qu'ils nous aident à défendre la cause de ces enfants et à trouver des solutions au problème, par exemple en envoyer quelques-uns se faire opérer en hôpital pédiatrique.

Maintenant que le pays entre dans une phase de reprise, un risque plane : que les efforts pour contrer les chamboulements dans le milieu de la chirurgie et reprendre le temps perdu avantagent les patients majeurs. Les enfants ne sont pas des petits adultes, et ne sont pas moins méritants. Ils ont des besoins chirurgicaux propres qui doivent aussi être soumis à un processus d'établissement des priorités dans le système de santé. 


\section{Références}

1. COVIDSurg Collaborative. Elective surgery cancellations due to the COVID-19 pandemic: global predictive modelling to inform surgical recovery plans. $\mathrm{Br} J$ Surg 2020 May 12 [Cyberpublication avant impression]. doi: 10.1002/ bjs.11746.

2. Table 4: Number, percentage ${ }^{\star}$ and average acute length of stay for top 10 highvolume inpatient surgeries by province/territory, HMDB, 2017-2018 [table]. Ottawa: Canadian Institute for Health Information. Accessible ici : www.cihi.ca/ sites/default/files/document/dad-hmdb-childbirth-quick-stats-2017-2018-fr.xlsx (consulté le 17 mai 2020).

3. Blair GK. Children are waiting for care and answers. CMAJ 2008;179:983-4.
4. Wright JG, Menaker RJ; Canadian Paediatric Surgical Wait Times Study Group. Waiting for children's surgery in Canada: the Canadian Paediatric Surgical Wait Times Project. CMAJ 2011;183:E559-64.

5. Paediatric surgical wait times report. FY 2018. Canadian Paediatric Decision Support Network. Accessible ici : ken.childrenshealthcarecanada.ca/xwiki/bin/ view/Canadian+Paediatric+Decision+Support+Network+\%28CPDSN\%29 (consulté le 17 juillet 2020).

6. Bai K, Liu W, Liu C, et al. Clinical analysis of 25 COVID-19 infections in children. Pediatr Infect Dis J 2020;39:e100-3.

7. Yuan $\mathrm{C}$, Zhu $\mathrm{H}$, Yang $\mathrm{Y}$, et al. Viral loads in throat and anal swabs in children infected with SARS-CoV-2. Emerg Microbes Infect 2020;9:1233-7.

\section{Intérêts concurrents : Aucun déclaré.}

Cet article a été révisé par des pairs.

Affiliations : Département de chirurgie, Université de la ColombieBritannique; Service de chirurgie pédiatrique, Hôpital pour enfants de la Colombie-Britannique, Vancouver, C.-B.

Membres des Pediatric Surgical Chiefs of Canada : Erik D. Skarsgard* MSc MD (chirurgie pédiatrique), Université de la Colombie-Britannique et Hôpital pour enfants de la Colombie-Britannique, Vancouver, C.-B.; Juan Bass† MD (chirurgie pédiatrique), Université d'Ottawa et Centre hospitalier pour enfants de l'est de l'Ontario, Ottawa, Ont.; Paul Beaudry† MD (chirurgie pédiatrique), Université de Calgary et Hôpital pour enfants de l'Alberta, Calgary, Alb.; Gerard Corsten† MD (otorhinolaryngologie et chirurgie cervico-faciale pédiatriques), Université Dalhousie et Centre de soins de santé IWK, Dalhousie, N.-É.; James Drake† MD (neurochirurgie pédiatrique), Université de Toronto et Hôpital SickKids, Toronto, Ont.; Mark Evans† MD (chirurgie pédiatrique), Université de l'Alberta et Hôpital pour enfants Stollery, Edmonton, Alb.; Jean-Pierre Farmer† MD (neuro- chirurgie pédiatrique), Université McGill et Hôpital de Montréal pour enfants, Montréal, Qc; Helene Flageole (présidente)† MD (chirurgie pédiatrique), Université McMaster et Hôpital pour enfants McMaster, Hamilton, Ont.; Richard Keijzer† MD (chirurgie pédiatrique), Université du Manitoba et Hôpital pour enfants de Winnipeg, Winnipeg, Man.; Elke Mau† MD (urologie pédiatrique), Université de la Saskatchewan et Hôpital pour enfants Jim-Pattison, Saskatoon, Sask.; Sandeep Mayer† MD (chirurgie pédiatrique), Université de Sherbrooke et Centre hospitalier de l'Université de Sherbrooke, Sherbrooke, Qc; Pascale Prasil† MD (chirurgie pédiatrique), Université Laval et Centre hospitalier de l'Université Laval, Sainte-Foy, Qc; David Price† MD (chirurgie pédiatrique), Université Memorial de Terre-Neuve et Centre de santé et de réadaptation pour enfants Janeway, St. John's, T.-N.-L.; Leslie Scott† MD (chirurgie pédiatrique), Université Western et Hôpital pour enfants du Centre des sciences de la santé de London, London, Ont.; Dominic Venne† MD (neurochirurgie pédiatrique), Université de Montréal et Centre hospitalier universitaire Sainte-Justine, Montréal, Qc. *Auteur; †Contributeurs.

Correspondance : Erik Skarsgard, eskarsgard@cw.bc.ca 